\title{
Microbiological Features of Upper Respiratory Tract Infections in Bulgarian Children for the Period 1998-2014
}

\author{
Raina Tzvetanova Gergova ${ }^{1}$, Guergana Petrova ${ }^{2}$, Stefan Gergov $^{3}$, Petko Minchev ${ }^{4}$, Ivan Mitov ${ }^{1}$, Tanya Strateva $^{1}$ \\ ${ }^{1}$ Department of Medical Microbiology, Medical University of Sofia School of Medicine, Sofia, Bulgaria \\ ${ }^{2}$ Pediatric Cilinic, UMHAT “Alexandrovska" Medical University of Sofia, Sofia, Bulgaria \\ ${ }^{3}$ Department of Otolaryngology, National Medical Center of Oncology, Sofia, Bulgaria \\ ${ }^{4}$ Pediatric Pulmonogy Clinic, USHATLD "Sveta Sofia”, Sofia, Bulgaria
}

Background: Across the globe, upper respiratory tract infections (URTIs) are the most prevalent cause of morbidity in childhood.

Aims: The aim of our study is to analyze the incidence and etiology of bacterial URTIs in Bulgarian children, as well as the increasing antimicrobial resistance to the most common etiologic agents over a period of 17 years. Study Design: Retrospective study.

Methods: The study material comprised the data from 4768 patients (aged 1-16 years) with URTI during the period from 1998-2014. Specific microbiology agent detection was performed by culture examination. Susceptibilities to the investigated pathogens were determined by the disk diffusion method and minimal inhibitory concentration according to the criteria of the Clinical and Laboratory Standards Institute (CLSI). Polymerase chain reaction was used to detect the presence of $\beta$-lactam resistance genes.

Results: We identified the following as the most common URTI bacterial pathogens: Streptococcus pneumoniae (40.94\%), Streptococcus pyogenes (34.16\%), Haemophilus influenzae (44.23\%), Moraxella catarrh- alis (39.19\%) and Staphylococcus aureus (23.88\%). In more than $70 \%$ of cases, a polymicrobial etiology was found. The most commonly affected individuals were pre-school-aged children, which accounted for more than $36 \%$ of all patients. During the study period, a dramatic increase in resistance to antibiotic agents was observed. The most frequent types of resistance were the enzymatic inactivation of penicillins and cephalosporins (close to $100 \%$ in staphylococci and moraxellae) and inducible macrolide-lincozamide resistance (about $20 \%$ of Gram-positive cocci).

Conclusion: Due to mandatory immunization against pneumococci and $H$. influenzae in Bulgaria and the vast expanding resistance to the most popular antimicrobial agents changes in the etiology of URTI have recently been noted. Regular analysis of this etiological dynamic and the antimicrobial resistance of respiratory pathogens is important for choosing the correct therapy and successful treatment.

Keywords: Upper respiratory tract infections, antimicrobial susceptibility
Globally, respiratory infections represent the most common cause of morbidity in children and often require antimicrobial therapy $(1,2)$ The typical onset is like a banal viral infection, but on the contrary from the adult patients where the infections are often self-limiting and rarely cause any complication, Upper respiratory tract infections (URTIs) in childhood have a specific characteristics - they frequently are with combined microbial etiology, with prolonged courses and quickly evolve into lung inflammatory complications. This secondary infection is usually a bacterial one originating from the conditionally pathogenic microorganisms composing the normal colonizing flora of nasopharynx (1-5).

The rapid development of bacterial resistance in recent years to the most popular antimicrobial agents has affected the etiology of respiratory infections, as well as recommendations regarding the initial approach to therapy (6-10). 
The aim of our study is to analyze the etiology and incidence of bacterial URTIs in Bulgarian children as well as the increasing antimicrobial resistance to the most common antibiotic agents for a period from 1998 to 2014 .

\section{MATERIALS AND METHODS}

\section{Patients}

The study material comprised the data from 4768 patients. The inclusion criteria that were met by all patients were: children with a clinical manifestation respiratory infection aged from 1 to 16 years; consulted with a certified pediatrician and an otorhinolaryngologist to prove the presence of least one of the following disease entities: rhinosinusitis, ethmoiditis, nasopharyngitis, pharyngotonsillitis, adenoiditis; without previous antimicrobial treatment for at least 7 days before microbiological testing. The tests were performed after obtaining a signed informed consent of the legal guardian. The patients with lower airways or lung involvement or with sepsis were excluded. There was no personal patient information in the database. The Hospital's Ethics Committee granted study approval. The therapy prescribed was as all the clinical practice and guidelines approved, according the current pathogen.

\section{Clinical material and methods}

For the analyzed period, we examined the following samples: from patients with rhinopharyngitis, tonsillopharyngitis, and adenoiditis: nasopharyngeal swab $(\mathrm{n}=2848)$; from patients with rhinosinusitis, ethmoiditis and rhinopharyngitis: nasal swab $(n=1625)$ or puncture liquid from sinus $(n=105)$. All samples were obtained before the first administration of antimicrobial therapy. A special procedure is used for obtaining of samples in children with epiglottitis (250) - the tongue is stretched far forward and thus the larynx rises up and the epiglottis opens. A sterile aspiration drain pad or swab is then taken for examination. All the samples were obtained by a specialist from the affected area and then sent to the laboratory in the corresponding transport medium.

The standard protocol for microbiological diagnostic follow-up and tests was followed in the laboratory. For isolation and detection of the rigorous etiologic bacterial agents we used routine nutrition media such as Blood agar base supplemented with 5\% sheep blood, MacConkey agar, Candida chrome agar and developed and implemented by our team selective media chocolate agar with vancomycin for moraxellae and haemophilae, for their identification, as well as a quantitative method for correct evaluation of the clinical significance of isolates (3). The cultivation of inoculated samples on different media was performed in aerobic and in microaerophilic atmosphere respectively for various pathogens $(3,7)$. For identification of the isolated clinical strains we used mainly the products and systems by Crystal BD BBL (BBL; Becton, Dickinson, Germany) and RapID System Remel Thermo Fisher Scientific (Remel, Thermo Fisher Scientific Remel Products; Santa Fe, USA). All bacterial isolates from the symptomatic children, before treatment, in a microbial number over the critical one for an infectious process $(\geq 100000 \mathrm{CFU} / \mathrm{mL})$, were considered as significant (3). The other indicative tests for bacterial infection such as C-reactive protein (CRP) and leukocytosis with granulocytosis (more than $77 \%$ ) in manual screens of differential blood smears had been positive as well. For CRP detection (positive test $6 \mathrm{mg} / \mathrm{L}$ or higher) was used serums collected by standard procedures and slide agglutination test C-Reactive protein (CRP) latex (BioSystems S.A.; Costa Brava, Barcelona, Spain).

Susceptibilities to investigated pathogens were determined by Kirby-Bauer disk-diffusion method. Due to the necessity for some peculiar cases and for a representative sample strain, minimal inhibitory concentration (MIC) according the criteria of CLSI were measured $(7,11)$. B-lactamase production in staphylococci, moraxellae and haemophilae was detected with Cefinase disc BD BBL, and by using polymerase chain reaction (PCR) B-lactam resistance genes were probed $(7,8,12)$. According the results from in vitro test for antimicrobial susceptibility recommendation for treatment was given and some conclusions for a correct empiric therapy were established.

\section{Statistical analysis}

The data were analyzed using Chi-square and Fisher's exact test for categorical variables. All analytical procedures were performed using Statistical Package for the Social Sciences (SPSS) for Windows, Version 16.0. (SPSS Inc.; Chicago, IL, USA). We considered $p$ values of $\leq 0.05$ to indicate statistical significance.

\section{RESULTS}

In $77 \%$ of cases, a specific bacterial etiology was confirmed. The bacterial spectrum of the URTI causes in children with different clinical entities is presented in Figure 1. The most often isolated bacterial etiological agents were S. pneumoniae (40.94\%), S. pyogenes (34.16\%), Staphylococcus aureus (S. aureus) (23.88\%), Haemophilus influenzae (H. influenza) (44.23\%) and Moraxella catarrhalis (M. catarrhalis) (39.19\%). The total is over $100 \%$ due to the many cases of different polymicrobial associations i.e. Streptococcus pneumoniae (S. pneumoniae) with $H$. influenzae and M. catarrhalis (12.26\%); S. pneumoniae with $H$. influenzae and Staphylococcus aureus (17.65\%); S. pyogenes with M. catarrhalis (10.81\%); H. influenzae with M. catarrhalis (8.31\%); Streptococ- 
cus pyogenes (S. pyogenes) with H. influenzae (3.49\%) (Figure 1). As is obvious from the figure, each disease has a unique etiologic profile. Statistically significant differences are found between etiology of epiglottitis and tonsillopharyngitis $(\mathrm{p}=0.001)$; epiglottitis and rhinopharyngitis $(\mathrm{p}=0.004)$; epiglottitis and rhinosinusitis $(\mathrm{p}=0.012)$; tonsillopharyngitis and ethmoiditis $(\mathrm{p}=0.000)$; tonsillopharyngitis and adenoiditis $(\mathrm{p}=0.023)$; adenoiditis and ethmoiditis $(\mathrm{p}=0.011)$; tonsillopharyngitis and rhinosinusitis $(\mathrm{p}=0.000)$; adenoiditis and rhinosinusitis $(\mathrm{p}=0.000)$; ethmoiditis and rhinopharyngitis $(\mathrm{p}=0.000)$; rhinosinusitis and rhinopharyngitis $(\mathrm{p}=0.000)$.

Table 1 shows the distribution according the patients' age and the URTI diagnosis (Table 1). Our results show, that the most affected age groups are from 1 to 6 years $(p<0.5)$, with extremely close frequency in the first two groups and the most common diagnoses are rhinopharyngitis, tonsillopharyngitis and adenoiditis.

The comparison in the resistance patterns of 1998 with that of 2003, 2009 and of 2014 for different pathogens may be seen in Figure 2 and Figure 3. Over the seventeen-year period, the regression of sensitivity to many antimicrobial drugs is detected. Overall antimicrobial susceptibility through the years statistically decreases from 1998 till 2014: for S. pneumoniae $(\mathrm{p}=0.010)$, S. pyogenes $(\mathrm{p}=0.000)$ and $H$. influenza $(\mathrm{p}=0.006)$, while the results for $M$. catarrhalis and $S$. aureus were not statistically significant with $\mathrm{p}=0.23$ and $\mathrm{p}=0.11$ respectively. Significant drop of susceptibility with $\mathrm{p}<0.05$ from 1998 till
2014 towards particular antimicrobials for each pathogen was found for: penicillin, ampicillin, amoxicillin/clavulanic acid, azithromycin, tetracycline, sulfamethoxazole/trimethoprim and clindamycin for S. pneumoniae; azithromycin, tetracycline and clindamycin for $S$. pyogenes; penicillin, ampicillin, azithromycin and tetracycline for $S$. aureus; ampicillin, cefuroxime and tetracycline for $H$. influenzae; ampicillin, cephalexin and cefuroxime for M. catarrhalis.

\section{DISCUSSION}

The most widespread bacterial agents causing rhinosinusitis and ethmoiditis were S. pneumoniae and Staphylococcus aureus, followed rarely by $H$. influenzae and $M$. catarrhalis, while isolates in the majority of cases involved various combinations (Figure 1). From the results it is noteworthy that in contrast to the infectious process of some other body systems, the URTI, particularly rhinosinusitis, ethmoiditis, rhinopharyngitis and adenoiditis most often (in more than $70 \%$ of cases) were with polymicrobial etiology. Similar results have also been reported in our earlier studies as well as other authors $(3,4,13,14)$.

The explanation may involve the fact that Gram-positive cocci form the most frequent type of nasal mucous coloniza-

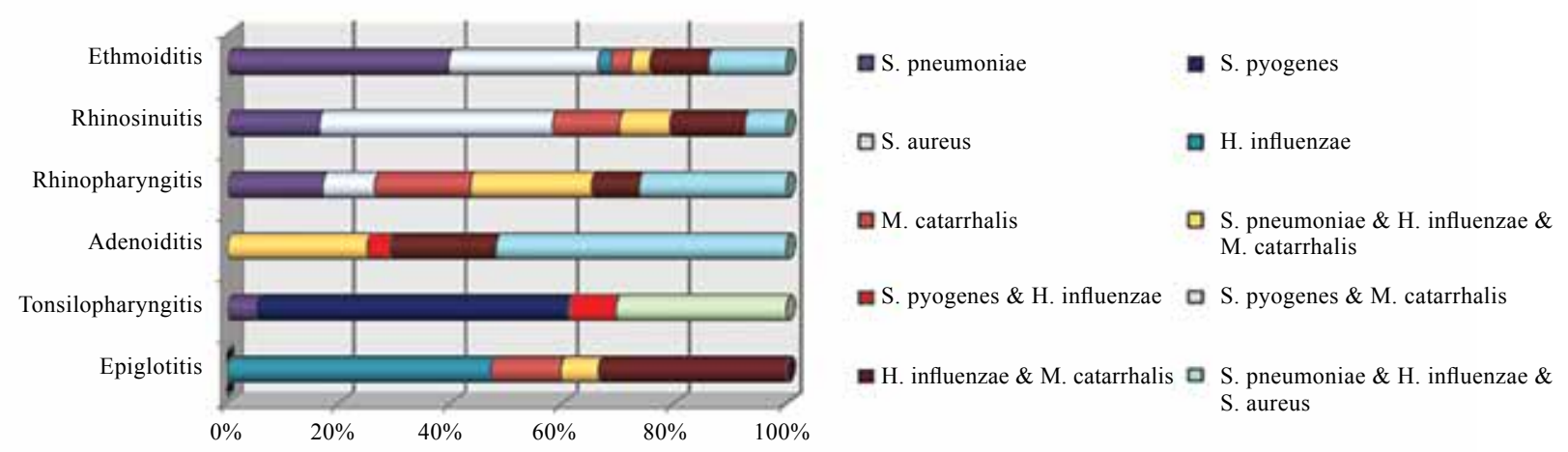

FIG. 1. Clinically significant isolates from children with URTI

TABLE 1. Age and disease entity distribution among 4768 patients with URTI

\begin{tabular}{|c|c|c|c|c|c|c|c|c|c|c|c|c|}
\hline \multirow{2}{*}{ Diagnosis } & \multicolumn{2}{|c|}{ Age $<3$ y } & \multicolumn{2}{|c|}{ Age $4-6$ y. } & \multicolumn{2}{|c|}{ Age $7-10$ y. } & \multicolumn{2}{|c|}{ Age $11-13$ y. } & \multicolumn{2}{|c|}{ Age $14-16$ y. } & \multicolumn{2}{|c|}{ Total } \\
\hline & Number & $\%+\mathrm{SD}$ & Number & $\%+\mathrm{SD}$ & Number & $\%+\mathrm{SD}$ & Number & $\%+\mathrm{SD}$ & Number & $\%+\mathrm{SD}$ & Number & $(\%+\mathrm{SD})$ \\
\hline Rhinopharyngitis & 890 & $18.67+1.11$ & 825 & $17.30+1.07$ & 136 & $2.85+0.47$ & 12 & $0.25+0.14$ & - & & 1863 & $39.07+1.39$ \\
\hline Rhinosinusitis & - & & - & & 34 & $0.71+0.24$ & 47 & $0.99+0.28$ & 56 & $1.17+0.31$ & 137 & $2.87+0.47$ \\
\hline Tonsillopharyngitis & 350 & $7.34+0.74$ & 414 & $8.68+0.80$ & 388 & $8.14+0.78$ & 295 & $6.19+0.68$ & 222 & $4.66+0.60$ & 1669 & $35.00+1.35$ \\
\hline Ethmoiditis & 309 & $6.48+0.13$ & 269 & $5.64+0.65$ & 20 & $0.42+0.18$ & - & & - & & 598 & $12.54+0.94$ \\
\hline Adenoiditis & 103 & $2.47+0.44$ & 135 & $3.24+0.50$ & 13 & $0.32+0.16$ & - & & - & & 251 & $5.27+0.63$ \\
\hline Epiglottitis & 94 & $2.25+0.42$ & 85 & $1.97+0.39$ & 45 & $1.08+0.29$ & 16 & $0.38+0.17$ & 10 & $0.24+0.14$ & 250 & $5.25+0.63$ \\
\hline Total & 1746 & $6.62+1.37$ & 1728 & $36.24+1.36$ & 636 & $13.34+0.97$ & 370 & $7.76+0.76$ & 288 & $6.04+0.68$ & 4768 & $100+0$ \\
\hline
\end{tabular}

URTI: upper respiratory tract infection; SD: standard deviation 
tion $(1,15)$. The biggest biodiversity was found in rhinopharyngitis and adenoiditis, where environ in $30 \%$ of the samples a triple polymicrobial combination was defined as respiratory infection agents namely $S$. pneumoniae, $H$. influenzae and $M$. catarrhalis. Only in tonsillopharyngitis predominantly a mono-infection was detected with the leading etiology role of $S$. pyogenes, in two third of the cases alone. The combined isolation of pyogenic streptococci and moraxellae had been already explained by the proven genetic mechanisms of $S$. pyogenes for a synergy with $M$. catarrhalis and their co-growth in cell cultures, i.e. the moraxellae potentates the adhesion of $S$. pyogenes to nasopharyngeal epithelium $(4,13)$.

For children with bacterial etiology of the epiglottis in $49 \%$ of cases $H$. influenzae was isolated, alone, in 13\% M. catarrhalis alone and in over $35 \%$ both agents in combination (Figure 1). A little known fact is that the most common microbial combination of causative agents, actually are exactly those two microorganisms in association, not only for epiglottitis but also for other URTI as adenoiditis and rhinopharyngitis in children, that have been confirmed by other authors through the last years (14).

Selective pressure from the common and not always well grounded use of macrolides in childhood leads to fast expansion of streptococcal strains with limited or absent susceptibility towards these antibiotics, which could lead to serious complications and sequels after infections caused by them (16-20). Confirmed data for appearance of new more virulent strains of $M$. catarrhalis with obligatory production of $\beta$-lactamase during the last years have been published even in Bulgaria $(8,12)$. Often mucous adhesion and pathogenic activity of one microorganism is enhanced in a presence of other species. This happens especially in cases with bacterial biofilm formation, where bacterial metabolism is amplified and thus the host immune defense system is more hampered by these more virulent bacterial species $(2,10)$. In such cases the infection process, from upper respiratory tract mucus, propagates outside the initial localization and leads to complications such as middle ear infections or pneumonia, in some cases even it can lead to bacteremia or infections of central nervous system (2,18,20-22).

Rhinopharyngitis and tonsillopharyngitis are predominantly in the age when the child is enrolled in kindergarten. While the first ones are mainly diagnosed for the younger children, tonsilopharyngitis are evenly distributed throughout the ages. Our data are supported by previous studies and could easily be explained by inefficiency of the local mucous defense in upper airways $(1-3,22,23)$. After the $6^{\text {th }}$ year of age, infections gradually decrease due to the sophistication of the immune system, and to the developing of adaptive immunity after initial contact with respiratory pathogens.
The basic mechanism leading to problematic resistance of staphylococci, moraxellae and haemophili towards $\beta$-lactams is the production of enzymes that destruct the active center of the antimicrobial agent (ß-lactam ring). In the year 2014 100\% of all samples of outpatient cases with $M$. catarrhalis and almost $90 \%$ from S. aureus, produce $\beta$-lactamase, which makes them resistant to aminopenicillins without inhibitor, and moraxellae are resistant also to all first generation cephalosporins (Figure 2c-3b). So far $\beta$-lactamase from M. catarrhalis, H. influenzae and $S$. aureus could be suppressed by the well-known inhibitors as sulbactam and clavulonic acid, but recently there were some reports for a broader spectrum of $\beta$-lactamase activity $(5,7,16)$.

High frequency of production of extracellular $\beta$-lactamase is accounted as an indirect virulence factor $(12,19)$. Whether any of these microorganisms is an independent etiologic agent or is isolated with other susceptible bacteria as co-infection, most commonly streptococci, due to the actions of these extracellular enzymes, the effect of penicillin or cephalosporin in the treatment is suppressed. This prevents the eradication of the main causative agents that are sensitive to B-lactam antibiotic and causes complications or development of chronic recurrent respiratory infection $(10,13)$.

The second more rare mechanism (the change of the penicillin-binding proteins) is the reason for resistance towards $B$ lactams in near $20 \%$ of Bulgarian isolates S. pneumoniae and $S$. aureus (Figure 2a-c). The latter has a strong negative effect on the actions of all penicillins and cephalosporins similar to the other countries in Europe and USA $(7,12,15,16)$. First reports for macrolide-resistant $S$. pyogenes are published in the nineties in the end of the $20^{\text {th }}$ century (19). Till 2003 we haven't isolated any resistant to macrolides $S$. pyogenes (Figure $2 b$ ). In analyzing the results in our study it was noted that the first strains with lower susceptibility towards macrolides were detected in the year 2004 - in the year 2014 almost 23\% of isolated in Bulgaria strains of $S$. pyogenes are with lower susceptibility towards the macrolides. Our results are similar to the results of multicenter study in Spain for an earlier period (2006-2007) (16). About $25 \%$ of $S$. pyogenes strains from macrolide resistant ones also express lower susceptibility towards lincosamides. Even higher macrolide-lincosamide resistance (over 30\%) is noted in 2014 in the S. pneumoniae and $S$. aureus strains, as it is presented in Figure 2. After the introducing of the new macrolides, suitable for respiratory infection therapy in childhood and their uncontrolled use for 10 years, now more than $1 / 3$ from isolates are resistant to macrolides mainly due to prevail of the induced mechanism of resistance to lincosamides. In recent years similar alarming data have been reported in many other European and non-European countries $(6,7,9,16,17,19,24)$. This fast growing resistance 

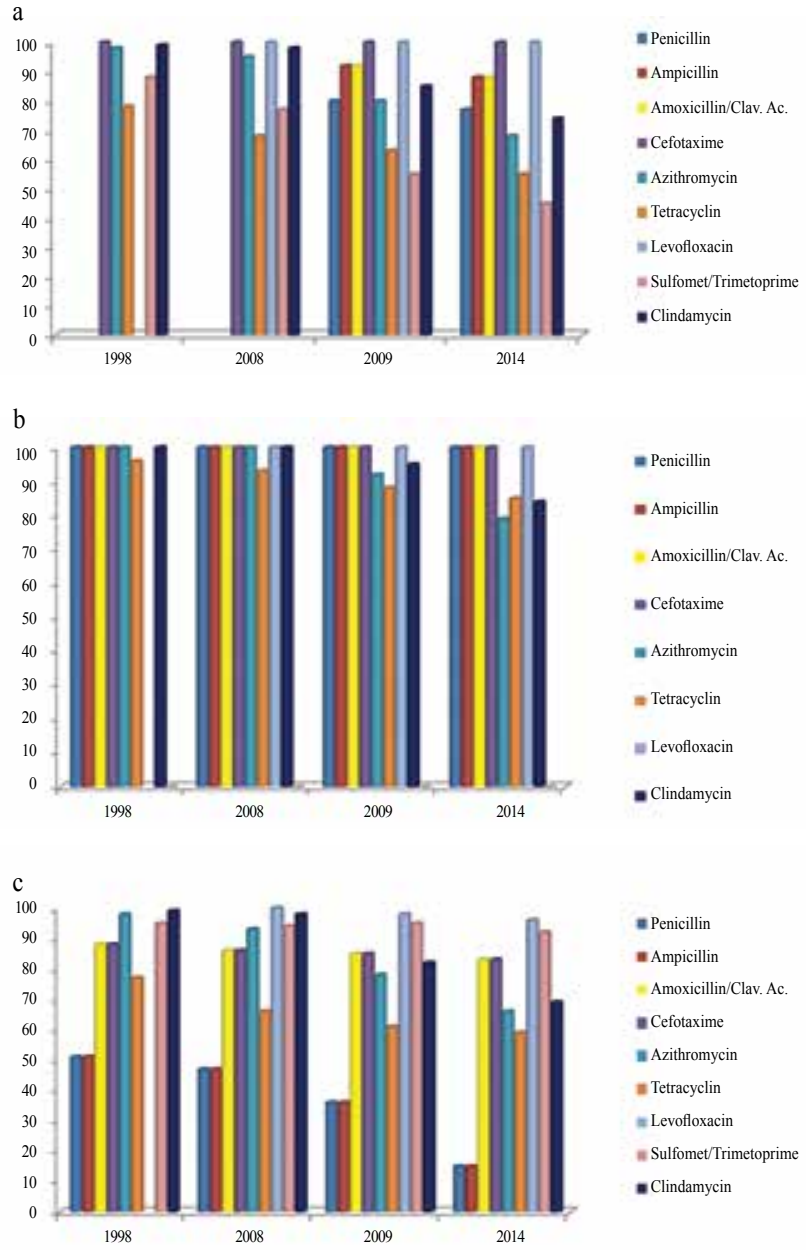

FIG. 2. a-c. Antimicrobial susceptibility of Gram-positive cocci presented in \%. S. pneumoniae (a); S. pyogenes (b); S. aureus (c).

due to the change of the action target in the last ten years has become a serious problem for therapy, particularly in combination with a change in penicillin-binding proteins because they are major classes of antimicrobial agents for the treatment of respiratory infections in children, and nowadays there has been reports for failures in the treatment of serious infections caused by multidrug-resistant strains $(6,9,15,19,20)$.

In conclusion, as the most common URTI pathogens, isolated mainly in polymicrobial associations, S. pneumoniae, $H$. influenzae, $M$. catarrhalis and $S$. aureus were identified; for tonsillopharyngitis, the predominant microorganism was $S$. pyogenes. URTI were most common in children of pre-school age. The most common resistance observed was to ß-lactams, due to the production of $\beta$-lactamase. Previously, this resistance could be bypassed by administereing amino-penicillin combined with a $\beta$-lactamase inhibitor, but this could be ineffective in the future. The most dynamically developing resistance in the last years has been that against macrolides in
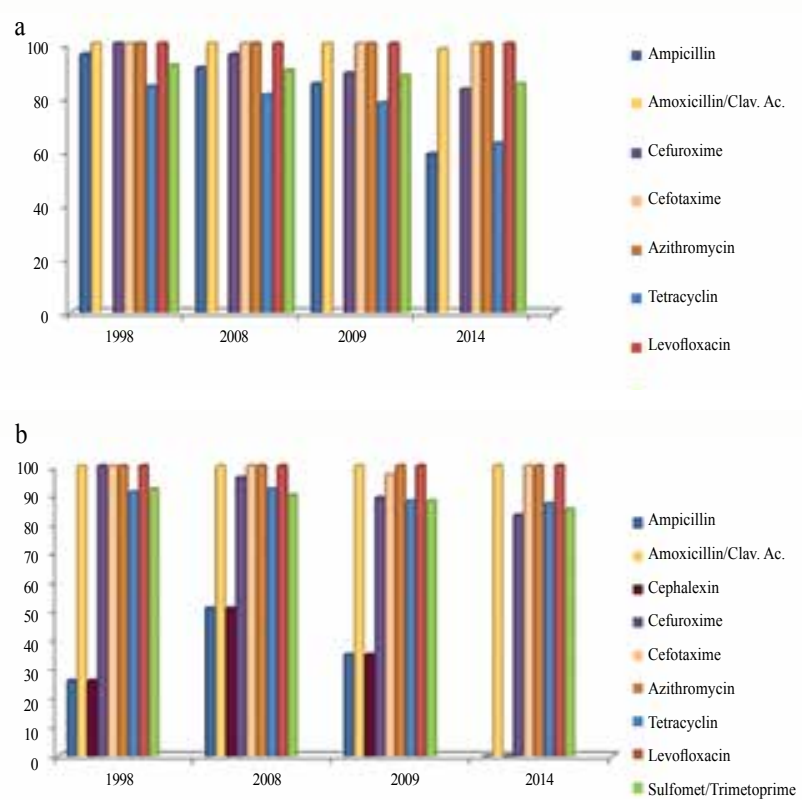

FIG. 3. a, b. Antimicrobial susceptibility of Gram-negatives, presented in $\%$. $\mathrm{H}$. influenzae (a); M. catarrhalis (b).

staphylococci and streptococci. This resistance often can be found crossed with low susceptibility towards other antimicrobial agents, mainly lincosamides. A combination of different resistance mechanisms in polyresistant strains has become more frequent in recent years and is extremely dangerous. The etiology of respiratory infections and the susceptibility to causative agents is changing and should be periodically monitored for correct antimicrobial policy and an accurate prognosis based on empirical therapy.

Ethics Committee Approval: Ethics committee approval for this study was received from the ethics committee of Medical University of Sofia School of Medicine.

Informed Consent: Written informed consent was obtained from parents of the patients who participated in this study.

Peer-review: Externally peer-reviewed.

Author contributions: Concept - R.G., T.S., S.G.; Design - R.G.; Supervision - I.M., T.S.; Resource - I.M., R.G.; Materials - S.G., G.P., P.M.; Data Collection and/or Processing - P.M., G.P., S.G.; Analysis and/or Interpretation - R.G., G.P.; Literature Search - R.G., G.P.; Writing - R.G., S.G.; Critical Reviews - R.G., I.M., P.M., G.P., S.G., T.S.

Conflict of Interest: No conflict of interest was declared by the authors.

Financial Disclosure: The authors declared that this study has received no financial support. 


\section{REFERENCES}

1. Kaplan SL, Hulten KG, Gonzalez BE, Hammerman WA, Lamberth L, Versalovic J, et al. Three-year surveillance of community-acquired Staphylococcus aureus infections in children. Clin Infect Dis 2005;40:1785-91. [CrossRef]

2. Dahlblom V, Söderström M. Bacterial interactions in the nasopharynx - Effects of host factors in children attending day-care centers. J Infect Public Health 2012;5:133-9. [CrossRef]

3. Gergova R, Mitov I, Minchev P. Criteria for isolation and evaluation of clinical significance of Moraxella (Branhamella) catarrhalis in respiratory tract samples. Modern medicine 2003;5:3-10. (in Bulgarian)

4. Verhaegh SJ, Flores AR, van Belkum A, Musser JM, Hays JP. Differential virulence gene expression of group A Streptococcus serotype M3 in response to co-culture with Moraxella catarrhalis. PLoS One 2013;8:62549. [CrossRef]

5. Hays JP. Moraxella catarrhalis: A mini review. J Pediatric Infect Dis 2009;4:211-20.

6. Setchanova LP, Alexandrova A, Mitov I, Nashev D, Kantardjiev T. Serotype distribution and antimicrobial resistance of invasive Streptococcus pneumoniae isolates in Bulgaria before the introduction of pneumococcal conjugate vaccine. $J$ Chemother 2012;24:12-7. [CrossRef]

7. Setchanova L, Gergova R, Mitov I. Antimicrobial susceptibility of respiratory tract isolates Streptococcus pneumoniae, Haemophilus influenzae and Moraxella catarrhalis. Modern Medicine 2004;6:3-10 (Bulgarian).

8. Gergova R, Markovska R, Mitov I. Antimicrobial resistance and production $\beta$-lactamase in Bulgarian clinical isolates Moraxella catarrhalis. Annals of Microbiology 2009;59:169-72. [CrossRef]

9. Patel M, Waites KB, Moser SA, Cloud GA, Hoesley CJ. Prevalence of inducible clindamycin resistance among communityand hospital-associated Staphylococcus aureus isolates. J Clin Microbiol 2006;44:2481-4. [CrossRef]

10. Budhani RK, Struthers JK. Interaction of Streptococcus pneumoniae and Moraxella catarrhalis: investigation of the indirect pathogenic role of $\beta$-lactamase-producing moraxellae by use of a continuous-culture biofilm system. Antimicrob Agents Chemother 1998;42:2521-6.

11. Clinical and Laboratory Standards Institute. Performance standards for antimicrobial susceptibility testing: 20th informational supplement. Wayne, Pa M100-S20, 2010.

12. Mitov IG., Gergova RT, Ouzounova-Raykova VV. Distribution of genes encoding virulence ompB2, ompCD, ompE, beta-lactamase and serotype in pathogenic and colonizing strains Moraxella catarrhalis. Arch of Med Res 2010;41:530-5. [CrossRef]
13. Lafontaine ER, Wall D, Vanlerberg SL, Donabedian H, Sledjeski DD. Moraxella catarrhalis coaggregates with Streptococcus pyogenes and modulates interactions of S. pyogenes with human epithelial cells. Infect Immun 2004;72:6689-93. [CrossRef]

14. Verhaegh SJ, Snippe ML, Levy F, Verbrugh HA, Jaddoe VWV, Hofman A, et al. Colonization of healthy children by Moraxella catarrhalis is characterized by genotype heterogeneity, virulence gene diversity and co-colonization with Haemophilus influenza. Microbiology 2011;157:169-78. [CrossRef]

15. Chavez-Bueno S, Bozdogan B, Katz K, Bowlware KL, Cushion $\mathrm{N}$, Cavuoti D, et al. Inducible clindamycin resistance and molecular epidemiologic trends of pediatric community-acquired methicillin-resistant Staphylococcus aureus in Dallas, Texas. Antimicrob Agents Chemother 2005;49:2283-8. [CrossRef]

16. Pérez-Trallero E, Martín-Herrero JE, Mazón A, García-Delafuente $\mathrm{C}$, Robles $\mathrm{P}$, Iriarte $\mathrm{V}$, et al. Antimicrobial resistance among respiratory pathogens in Spain: latest data and changes over 11 years (1996-1997 to 2006-2007). Antimicrob Agents Chemother 2010;54:2953-9. [CrossRef]

17. Jacobs MR, Johnson CE. Macrolide resistance: an increasing concern for treatment failure in children. Paediatr Infect Dis 2003;22(Suppl 8):131-8. [CrossRef]

18. Waterer GW, Wunderink RG, Jones CB. Fatal pneumococcal pneumonia attributed to macrolide resistance and azithromycin monotherapy. Chest 2002;118:1839-40. [CrossRef]

19. Dundar D, Sayan M, Tamer GS. Macrolide and tetracycline resistance and emm type distribution of Streptococcus pyogenes isolates recovered from Turkish patients. Microb Drug Resist 2010;16:279-84 [CrossRef]

20. Sowerby LJ, Hussain Z, Husein M. The epidemiology, antibiotic resistance and post-discharge course of peritonsillar abscesses in London, Ontario. J Otolaryngol Head Neck Surg 2013;42:5. [CrossRef]

21. Turel O, Yıldırım C, Yılmaz Y, Kulekci S, Akdaş F, Bakır M. Clinical characteristics and prognostic factors in childhood bacterial meningitis: a multicenter study. Balkan Med J 2013;30:804. [CrossRef]

22. Watt JP, Wolfson LJ, O’Brien KL, Henkle E, Deloria-Knoll M, McCall N, et al. Burden of disease caused by Haemophilus influenzae type $\mathrm{b}$ in children younger than 5 years: global estimates. Lancet 2009;374:903-11. [CrossRef]

23. Muge OA, Zeynep B, Cem A. Carriage rate of Haemophilus influenzae among preschool children in Turkey. Jpn J Infect Dis 2007;60:179-82.

24. Sirekbasan L, Gönüllü N, Sirekbasan S, Kuşkucu M, Midilli K. Phenotypes and genotypes of macrolide-resistant streptococcus pneumoniae. Balkan Med J 2015;32:84-8. [CrossRef] 